\title{
The influence of network structure on neuronal network dynamics
}

\author{
Duane Q Nykamp \\ From Twenty First Annual Computational Neuroscience Meeting: CNS*2012 \\ Decatur, GA, USA. 21-26 July 2012
}

We investigate the influence of network structure on the dynamics of neuronal networks, with a focus on the emergence of synchronous oscillations. Network structure is specified using the framework of second order networks, a network model that captures second order statistics (correlations) among the connections between neurons. We demonstrate that the frequency of a chain motif in the network plays a crucial role in influencing network dynamics, not only modulating the emergence of synchrony but also possibly increasing the range of possible network behaviors.
Published: 16 July 2012

doi:10.1186/1471-2202-13-S1-P79

Cite this article as: Nykamp: The influence of network structure on neuronal network dynamics. BMC Neuroscience 2012 13(Suppl 1):P79.

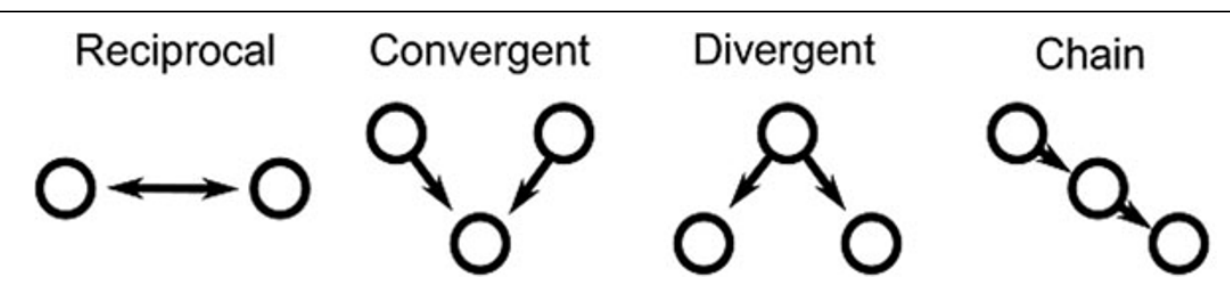

Figure 1 The four second order edge motifs of reciprocal, convergent, divergent, and causal connections. The frequency of these motifs determine the second order statistics, or correlations, among the network connections.

Correspondence: nykamp@umn.edu

School of Mathematics, University of Minnesota, Minneapolis, MN USA 55455

(c) 2012 Nykamp; licensee BioMed Central Ltd. This is an Open Access article distributed under the terms of the Creative Commons 